\title{
FINANCIAMIENTO Y DEMANDA DE EDUCACIÓN SUPERIOR EN COLOMBIA (PERIODO 1990-2005)
}

\section{Introducción ${ }^{4}$}

La educación genera beneficios individuales y sociales, representados no sólo en el aumento de la remuneración y el crecimiento de la economía, sino que también augura mejor calidad de vida para las personas que tienen acceso a ella. La literatura de crecimiento económico ha demostrado que la educación es uno de los mayores determinantes del crecimiento de un país ${ }^{5}$. Se ha observado que los países industrializados tienen un alto porcentaje de su población con acceso a niveles de estudio superiores a los observados en los países en vías de desarrollo. Por ejemplo, datos de 2004 indican que el porcentaje con acceso a la educación terciaria en los países de altos ingresos es del 66,7\%, mientras que para Latinoamérica esta cifra es de sólo el 28,6\% (Kapur y Megan Crowley, 2008). El gráfico 1 nos muestra datos de matrícula por año en entidades de educación terciaria y muestra la diferencia entre regiones del mundo.

4 Este artículo es parte del trabajo de investigación desarrollado en el Grupo de Economía y Empresa de la Universidad EAFIT.

5 Algunos artículos para destacar en este sentido son Barro, 1997; Barro y Lee, 1993; Benhabib y Spiegel, 1994; Easterly, 1981; Krueger y Lindhah, 2001, y Temple, 2001. 
Gráfico 1. Matrícula en educación terciaria (Unesco, 20/20)

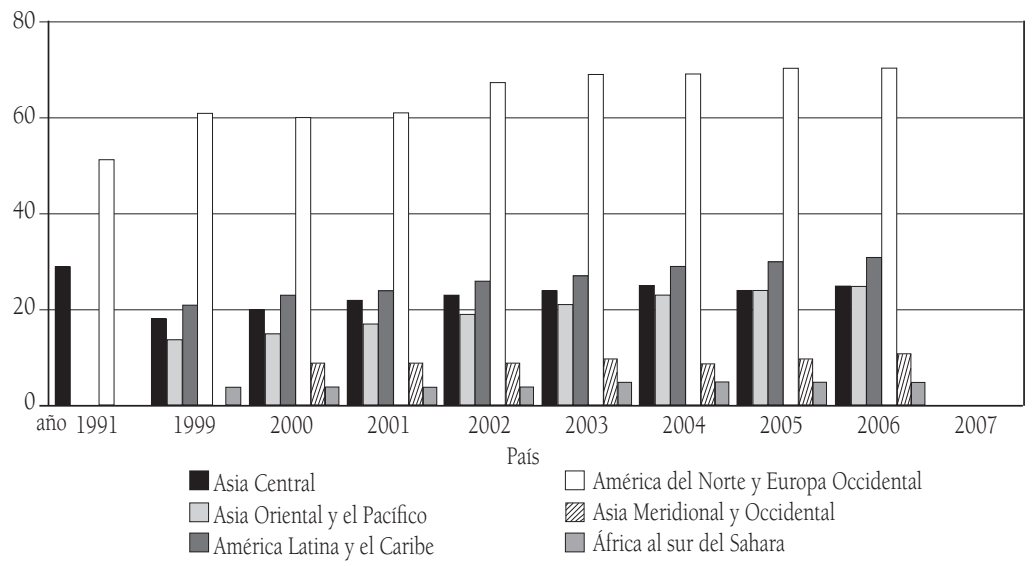

Fuente: 20/20 Unesco.

Dada su importancia, la literatura abunda en estudios sobre el impacto de la educación en el crecimiento económico de los países en vía de desarrollo. Sin embargo, un aspecto clave en el acceso a la educación es el financiamiento de la misma; la experiencia indica que los países en desarrollo deben abogar por mayores niveles de educación, pero la pregunta es sobre los determinantes económicos y financieros que permitan un incremento en el acceso. En particular, surge la pregunta de cómo financiar la educación, especialmente en el nivel superior ${ }^{6}$.

En el caso colombiano, el gobierno (entiéndase el sector público) no tiene la suficiente capacidad para generar el acceso general a este nivel educativo. Por esto, el sector privado ha buscado llenar el vacío a través del mecanismo del financiamiento individual de la educación. Pero, hasta ahora, el impacto del financiamiento (pública o privada) en la demanda por educación continúa siendo una pregunta de tipo empírico.

Para la presente investigación se parte del supuesto de que el individuo realiza un análisis de costo-beneficio en su proceso de

6 La educación superior es el nivel posterior a la educación media vocacional (seis años de educación primaria y seis años de educación secundaria). Se ofrece a quienes acreditan la calidad de bachiller y conduce a la obtención de títulos en las siguientes modalidades: formación intermedia profesional, formación tecnológica, formación universitaria y formación de posgrado. 
toma de decisiones. Es decir, la educación es vista como una inversión cuyo retorno debe ser mayor o igual a la tasa de rendimiento de las inversiones alternativas en el mercado. En este sentido, el objetivo es identificar la relación entre la demanda por educación superior (ES) y variables relacionadas a su financiamiento. En particular, se analiza cómo el financiamiento público y privado y las señales del mercado laboral explican las matrículas en ES en tres regiones de Colombia: Bogotá, Cali y Medellín. Este constituye un tema de gran relevancia debido a la importancia que estos niveles educativos tienen en la economía de un país. Además del financiamiento, tanto estatal como privado, se tienen en cuenta los demás factores que influyen en la decisión del individuo a la hora de demandar educación terciaria.

Se realizó un análisis empírico en el cual se estudió la situación para tres de las principales regiones de Colombia -Bogotá, Cali y Medellín-durante el periodo 1990-2005. Se estimó un modelo Datos de Panel que incluye diferentes determinantes financieros de la matrícula universitaria. Se consideraron como variables explicativas la tasa de interés del crédito educativo, los desembolsos de crédito educativo, el ingreso promedio de las personas que alcanzan el estudio universitario y de los que alcanzan el estudio técnico, el porcentaje del gasto público en relación con el producto interno bruto $^{7}$ y el desempleo regional ${ }^{8}$.

La literatura sobre el acceso a la educación y su impacto en el desarrollo económico es abundante (Krueger y Lindhah, 2001; Stevens y Weale, 2003); sin embargo, los estudios que se enfocan en el problema sobre el acceso a la ES y los recursos financieros y económicos son escasos, especialmente en países en vía de desarrollo $y$, en particular, en el caso colombiano ${ }^{9}$. El presente estudio ofrece una contribución en este sentido y, adicionalmente, una reflexión de tipo académico y para el desarrollo de políticas públicas. Los resultados indican que incrementos de la tasa de interés promedio de los créditos

7 El gasto público está dirigido a fomentar oferta en el sistema educativo.

8 Existen múltiples sesgos de selección. En particular una limitación de nuestro estudio es que nos es difícil cruzar umbrales como, por ejemplo, completar la educación secundaria y la rendición (primero) y luego aprobación de eventuales pruebas de admisión a las distintas casas de estudio. Por otro lado, puede haber un sesgo por parte de quienes otorgan los créditos y que en nuestro estudio nos es difícil diferenciar.

9 Uno de los pocos estudios encontrados para el caso de Colombia es el de Sánchez (2008). 
educativos afectan de forma negativa la demanda de la matrícula universitaria en ES en las ciudades de análisis y los ingresos de las personas con formación profesional tienen un impacto positivo.

El documento está organizado de la siguiente forma. En la sección dos se presenta un contexto de la demanda educativa y financiamiento de la educación para Colombia. En la sección tres se expone el marco conceptual y empírico y se presentan los resultados del modelo. Finalmente, en la sección cuarta se exponen las conclusiones.

\section{Demanda educativa y financiamiento en el contexto colombiano}

En Colombia el sistema de ES se encuentra organizado por la Ley 30 de 1992, emanada de la Presidencia de la República. La dirección y control del sistema corresponde al Gobierno Nacional, el cual delega en el Instituto Colombiano para el Fomento de la Educación Superior -ICFES- la acción de promover y coordinar el desarrollo del sector.

La oferta de ES en Colombia es predominantemente privada ${ }^{10}$. En 1990 había 242 instituciones, de las cuales el 30,6\% eran públicas y 69,4\% privadas. En 1999 las instituciones de educación superior (IES) del país aumentaron a 281. Las instituciones de origen público alcanzaron el 31,3\% y las privadas 68,6\%. En 2004 el total de IES eran $326,33 \%$ públicas y $67 \%$ privadas. En general, el crecimiento de las IES de 1990 a 2004 fue de 35\%, lo cual muestra un aumento significativo en la disponibilidad de oferta educativa en Colombia.

10 De acuerdo con el artículo 16 de la Ley 30 de 1992 las instituciones de educación superior se clasifican en: instituciones técnicas profesionales (ofrecen programas de formación en ocupaciones de carácter operativo e instrumental), instituciones tecnológicas (formación en ocupaciones, formación académica en disciplinas y programas de especialización en sus respectivos campos de acción), instituciones universitarias (facultadas para adelantar programas de formación en ocupaciones, programas de formación académica en profesiones o disciplinas y programas de especialización), universidades (reconocidas como tales y las que acrediten su desempeño con criterio de universalidad en: investigación científica o tecnológica; formación académica en profesiones o disciplinas y producción, desarrollo y transmisión del conocimiento y de la cultura universal y nacional. Están facultadas para ofrecer y realizar programas de formación en ocupaciones, profesiones o disciplinas, programas de especialización, maestrías, doctorados y posdoctorados) (Ministerio de Educación, 2002). 
Gráfico 2. Colombia. Crecimiento de las instituciones de educación superior por carácter académico (1990-2004)

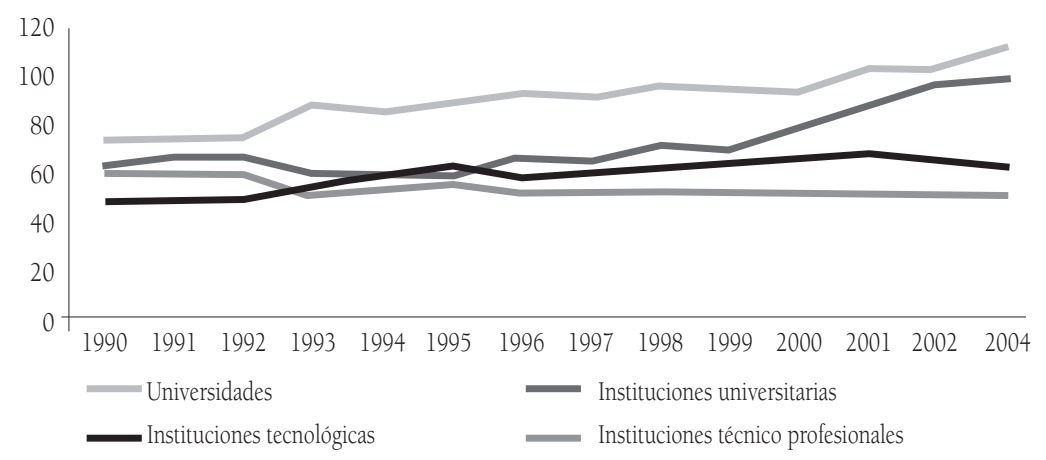

Fuente: Estadísticas de educación superior. Ministerio de Educación Superior de Colombia-MEN-. Nota: no fue posible encontrar datos para el año 2003.

Gráfico 3. Colombia. Número de instituciones de educación superior (1990-2004)

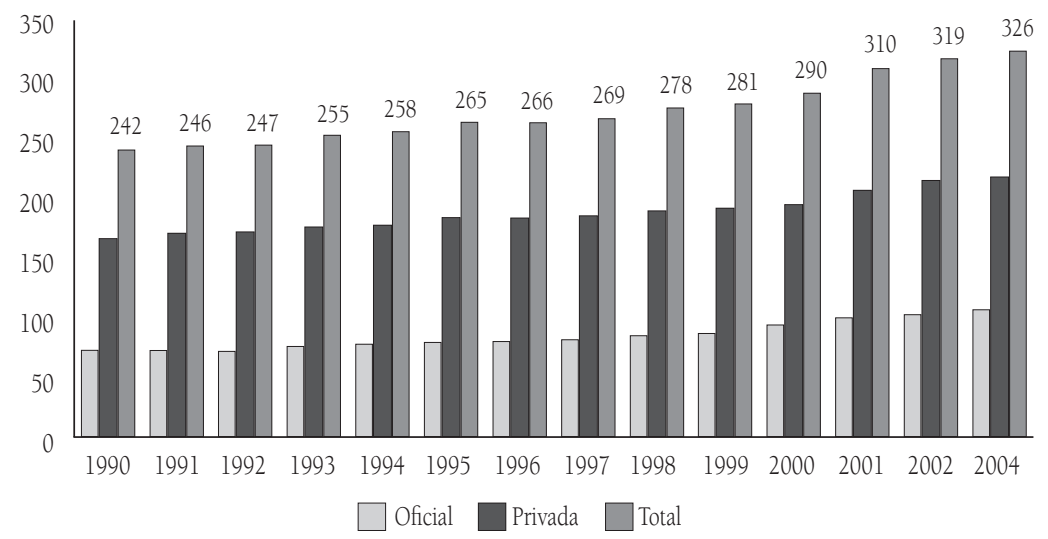

Fuente: Estadísticas de educación superior. Ministerio de Educación Superior de Colombia-MEN-. Nota: no fue posible encontrar datos para el año 2003.

La demanda en ES, según el Ministerio de Educación Nacional, se encuentra conformada por aquellos individuos que han obtenido el título de bachiller, prerrequisito indispensable para el ingreso a este nivel educativo. Se puede dimensionar la demanda a ES considerando toda la población colombiana con título de bachiller, lo cual identifica la demanda potencial ${ }^{11}$.

11 Además de otros ángulos de referencia, como las restricciones impuestas por las pruebas de admisión, restricciones geográficas y financieras. 
En Colombia hay una concentración de la matrícula nacional de educación superior en Bogotá y en los departamentos de Antioquia, Atlántico y Valle del Cauca, con 74\% para el total de la matrícula de 2005. La demanda por educación se enfrenta con la capacidad de oferta de las instituciones, es decir, los cupos disponibles ${ }^{12}$. En Colombia éstos crecieron en un 257\% entre 1990 y 2002, lo cual evidencia un aumento significativo. El crecimiento de los primíparos ${ }^{13}$ fue del $88 \%$ y de los matriculados en primer curso ${ }^{14}$ el $100 \%$ para el mismo periodo; esto muestra una brecha significativa entre el crecimiento de la oferta y la demanda por ES en el país.

Gráfico 4. Colombia. Oferta educativa (cupos) y demanda educativa (Primíparos y matriculados en primer curso) (1990-2002)

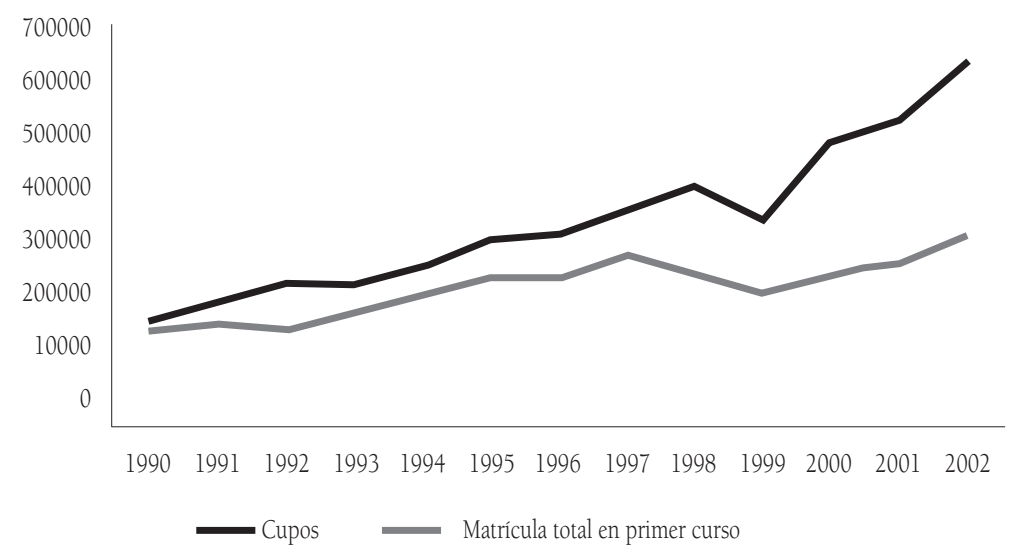

Fuente: Ministerio de Educación Superior de Colombia -MEN-.

12 Cupos: capacidad de oferta de las instituciones para atender la demanda de ingreso (primíparos) en un determinado programa académico.

13 Primíparos: aspirantes que, una vez son admitidos en una institución, inician su formación académica en el primer semestre de un programa o carrera, sin ser repitentes ni transferidos (Ministerio de Educación, 2002).

14 Matrícula total en primer curso: alumnos matriculados en el primer semestre académico de un programa. Se incluyen los primíparos, repitentes, transferencias, reintegros y demás formas de vinculación de personas que pertenecieron a algún nivel de la ES. No incluye alumnos del nivel introductorio a distancia, de cursos preuniversitarios o de nivelación en posgrado (Ministerio de Educación, 2002). 
El financiamiento de la ES en Colombia es diferente de acuerdo con su naturaleza. El Estado financia exclusivamente las IES públicas ${ }^{15}$, mientras las privadas se financian principalmente por el pago de matrículas y acuden a financieras mixtas o privadas para acceder a créditos de fomento como la Financiera de Desarrollo Territorial S.A. (FINDETER) ${ }^{16}$ y el Fondo de Desarrollo de la Educación Superior $(\text { FODESEP })^{17}$.

Para el financiamiento del pago de matrícula de estudiantes, el Instituto Colombiano de Crédito y Estudios Técnicos en el Exterior $(\text { ICETEX })^{18}$ otorga créditos educativos, con recursos propios o de terceros, a la población con menores posibilidades económicas y buen desempeño académico. En 2000, el 6\% de la población matriculada tuvo financiamiento con recursos del ICETEX (de las 26.482 solicitudes de crédito presentadas para pregrado en el país se financiaron 12.184); los demás estudiantes se financiaron con recursos propios o de las familias y con créditos a través de bancos, corporaciones y/o las instituciones educativas.

15 El financiamiento de la educación pública en Colombia tiene origen en el situado fiscal, el impuesto a la venta o valor agregado (IVA), los aportes Nacionales, los auxilios a las entidades territoriales, los aportes territoriales y demás transferencias. Los gastos de la educación se dividen en tres categorías: gastos de funcionamiento, gastos generales y gasto de operación comercial.

16 FINDETER realiza operaciones de redescuento que consiste en entregar recursos a un establecimiento de crédito o entidad financiera debidamente autorizada por ley para intermediar, con el objeto de financiar operaciones de crédito o de leasing, a cambio de cesión de garantías suficientes con el fin de ayudar al financiamiento e inversión en sectores para mejorar el desarrollo del país como: sector de agua, transporte, puentes fluviales e instituciones de educación, tanto públicas como privadas. Para más información ver. www.findeter.gov.co

17 El Fondo de Desarrollo de la Educación Superior, FODESEP, es una entidad de economía mixta, de derecho privado, sin ánimo de lucro, de interés social, organizada bajo los principios de la economía solidaria, creada por la Ley 30 de 1992 (artículos 89 al 91) y reglamentada por el Decreto Ley 2905 de 1994 que definió su funcionamiento, conformación y garantizó su desarrollo y el ejercicio pleno de sus actividades, así como el establecimiento de su vinculación al Ministerio de Educación Nacional y la inspección y vigilancia gubernamental de la Superintendencia de la Economía Solidaria. FODESEP tiene como objetivos generales promover el financiamiento de proyectos específicos de las instituciones y plantear y promover programas y proyectos para beneficio de las IES en concordancia con su desarrollo académico. Para más información ver http://www.fodesep.gov.co/nuevo/

18 ICETEX es una entidad estatal que promueve la ES a través del otorgamiento de créditos educativos a la población con menores posibilidades económicas y buen desempeño académico. Su objetivo es también facilitar el acceso a las oportunidades educativas que brinda la comunidad internacional. Para más información ver http://www.icetex.gov.co/ 
Uno de los créditos educativos enfocados a mejorar el acceso a la ES es el programa de Acceso con Calidad a la Educación Superior (ACCES). Este programa busca beneficiar a estudiantes de escuelas normales superiores, programas técnicos, tecnológicos y universitarios. El 45\% de los créditos ACCES son para Bogotá, Antioquia y Valle del Cauca; el resto se reparte en los demás departamentos del país con un financiamiento de hasta $75 \%$ de la matrícula y desde uno hasta cinco salarios mínimos legales vigentes para sostenimiento.

Tabla 1. Colombia. Beneficiarios Crédito ACCES 2003-2006

\begin{tabular}{|l|r|r|r|r|r|}
\hline Departamento & $\begin{array}{c}\text { Beneficiarios } \\
2003\end{array}$ & $\begin{array}{c}\text { Beneficiarios } \\
2004\end{array}$ & $\begin{array}{r}\text { Beneficiarios } \\
2005\end{array}$ & $\begin{array}{c}\text { Beneficiarios } \\
2006\end{array}$ & Participación \\
\hline Amazonas & 26 & 14 & 95 & 72 & $0.12 \%$ \\
\hline Antioquia & 1.802 & 1.750 & 1.251 & 1.350 & $8.55 \%$ \\
\hline Arauca & 109 & 60 & 42 & 104 & $0.52 \%$ \\
\hline Atlántico & 1.561 & 2.646 & 1.807 & 2.818 & $7.41 \%$ \\
\hline Bogotá, D.C. & 5.898 & 6.209 & 6.070 & 6.505 & $27.98 \%$ \\
\hline Bolívar & 953 & 946 & 840 & 1.525 & $4.52 \%$ \\
\hline Boyacá & 495 & 326 & 536 & 890 & $2.35 \%$ \\
\hline Caldas & 435 & 255 & 221 & 339 & $2.06 \%$ \\
\hline Caquetá & 112 & 81 & 99 & 197 & $0.53 \%$ \\
\hline Casanare & 13 & 26 & 136 & 262 & $0.06 \%$ \\
\hline Cauca & 358 & 217 & 214 & 359 & $1.70 \%$ \\
\hline César & 461 & 357 & 329 & 535 & $2.19 \%$ \\
\hline Chocó & 307 & 104 & 85 & 99 & $1.46 \%$ \\
\hline Córdoba & 423 & 387 & 256 & 446 & $2.01 \%$ \\
\hline Cundinamarca & 542 & 798 & 643 & 1.049 & $2.57 \%$ \\
\hline Guainía & 7 & 1 & 1 & 1 & $0.03 \%$ \\
\hline Guaviare & 15 & 1 & 3 & 18 & $0.07 \%$ \\
\hline La Guajira & 378 & 382 & 303 & 390 & $1.79 \%$ \\
\hline Huila & 391 & 252 & 307 & 779 & $1.85 \%$ \\
\hline Magdalena & 490 & 416 & 358 & 555 & $2.32 \%$ \\
\hline Meta & 190 & 146 & 166 & 331 & $0.90 \%$ \\
\hline Nariño & 766 & 467 & 500 & 1.249 & $3.63 \%$ \\
\hline Norte Santander & 675 & 554 & 450 & 482 & $3.20 \%$ \\
\hline Putumayo & 107 & 67 & 74 & 391 & $0.51 \%$ \\
\hline Quindío & 244 & 167 & 111 & 284 & $1.16 \%$ \\
\hline Risaralda & 269 & 221 & 167 & 345 & $1.28 \%$ \\
\hline San Andrés & 29 & 17 & 30 & 49 & $0.14 \%$ \\
\hline Santander & 1.354 & 1.446 & 1.053 & 1.626 & $6.42 \%$ \\
\hline Sucre & 312 & 282 & 242 & 472 & $1.48 \%$ \\
\hline Tolima & 653 & 414 & 467 & 756 & $3.10 \%$ \\
\hline Valle del Cauca & 1.691 & 1.740 & 1.406 & 2.476 & $8.02 \%$ \\
\hline Vaupés & 7 & 1 & 4 & 2 & $0.03 \%$ \\
\hline Vichada & 7 & 11 & 15 & 10 & $0.03 \%$ \\
\hline Total & 21.080 & 20.761 & 18.281 & 26.766 & $100.00 \%$ \\
\hline & & & & & \\
\hline
\end{tabular}

Fuente: Instituto Colombiano de Crédito y Estudios Técnicos en el Exterior - ICETEX, 2006. 
El número de desembolsos por semestre ha pasado de 9.408, en el segundo semestre del 2003, a 70.758 en 2007, es decir, se ha incrementado en $652 \%$ en cinco años. Esto muestra unos recursos significativos invertidos en formación profesional, que se espera se vea reflejado en un aumento en demanda por ES. El 79\% de los estudiantes solicitan el crédito para los tres primeros semestres, el $17,1 \%$ para los semestres cuarto al séptimo y tan sólo el 3,9\% para semestres superiores.

Gráfico 5. Colombia. Número de desembolsos por semestre 2003-2007

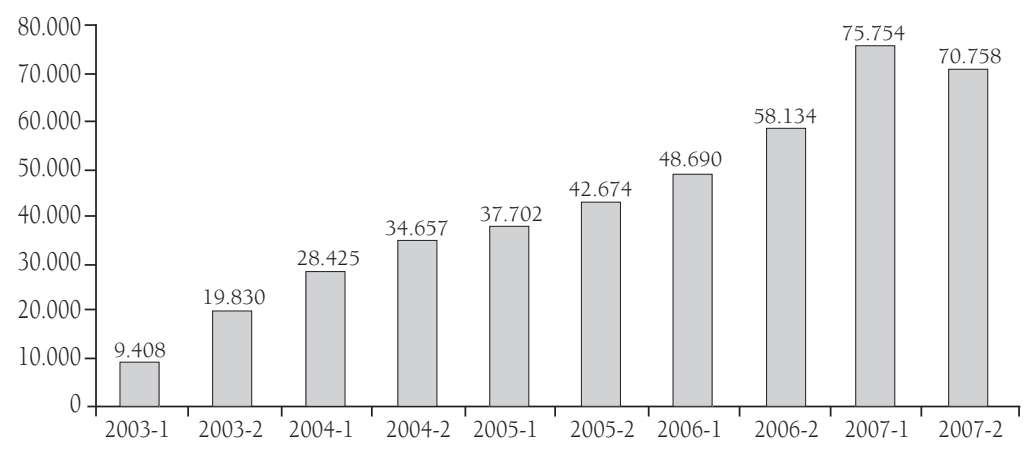

Fuente: Sánchez, 2008. ICETEX, Crédito Acces.

Gráfico 6. Colombia. Número de beneficiarios según número de desembolsos

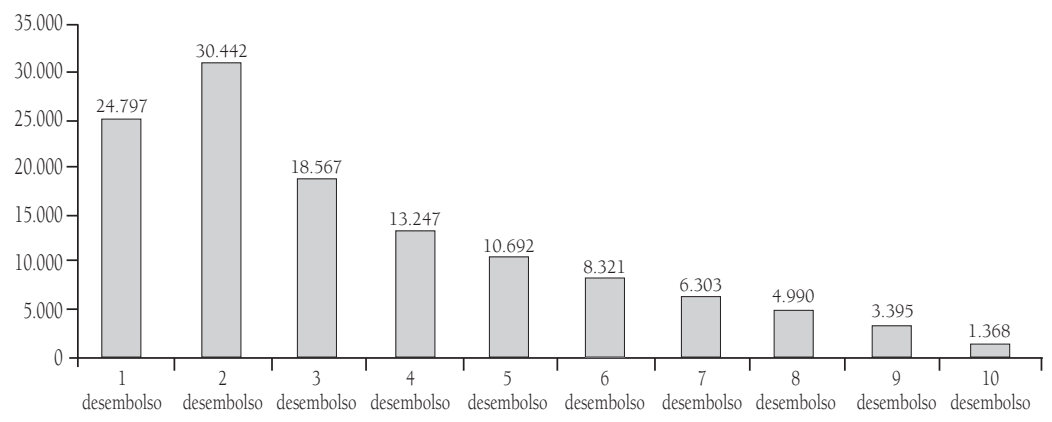

Fuente: Sánchez, 2008. ICETEX, Crédito Acces.

Las instituciones que ofrecen créditos educativos enfrentan el dilema de repago de la deuda. Existe incertidumbre acerca de las futuras fuentes de repago; es decir, iel deudor va a pagar con las ganancias futuras generadas en el mercado laboral? Es necesario, 
tanto para las instituciones como para los agentes, comparar los salarios reales proyectados con la inversión en el crédito bancario. Actualmente, debido a los riesgos, el sistema de financiamiento de la educación ha sido insuficiente. Por esto se requiere un trabajo mancomunado entre el Estado, las IES y el sistema financiero.

\section{Modelo conceptual y empírico para el análisis del impacto del financiamiento y la demanda de ES}

En esta sección se discuten consideraciones conceptuales y empíricas para el análisis de la demanda por ES. Primero, discutimos brevemente el estado del arte en el tema de la educación y sus fuentes de financiamiento y también abordamos los aspectos teóricos que determinan el modelo empírico. La segunda parte de esta sección introduce el modelo que se estima y la tercera discute sus resultados.

\subsection{Consideraciones teóricas y conceptuales}

En los estudios de financiamiento de la ES, Canton y De Jong (2005) investigaron el rol de los factores económicos en la decisión de entrar a la universidad. En ésta la educación es tomada como una inversión; es decir, los estudiantes potenciales toman en cuenta el retorno de la inversión de estudiar. Los resultados del ejercicio econométrico indicaron que los estudiantes no respondían a los costos de matrícula. Sin embargo, variables como el apoyo financiero (préstamos, becas, entre otros), el premium de educación (el retorno a la educación medido como las ganancias en el mercado de trabajo) y el salario actual eran determinantes importantes para el acceso a la ES en el caso de Holanda. Bajo el análisis sobre las formas de ES, McPherson y Schapiro (2006) concluyen que las becas al mérito o subsidios no son necesarios porque suscitan fuertes presiones en los estudiantes y atentan contra el principio de que la educación debe cobrarse de acuerdo con la capacidad familiar para pagar.

Ionescu (2006), basada en el modelo del ciclo vital de BenPorath -ganancias y capital humano-, mide el efecto y la decisión de invertir en educación universitaria de acuerdo con el valor presente 
de los ingresos futuros. Los principales postulados son que el capital humano es productivo sólo hasta la graduación y que la inversión en educación es riesgosa porque la tasa de interés es incierta.

La decisión por parte de los jóvenes de ingresar a la ES genera riesgos. Forero y Bardey (2008) asumen que cuando los estudiantes empiezan a estudiar una carrera es relativamente difícil para ellos predecir exactamente cómo será el mercado laboral cuando finalicen sus estudios. También la falta de liquidez durante el periodo de formación es otra característica que acentúa el riesgo en la decisión de educarse.

Se podría pensar a priori, de acuerdo con Forero y Bardey (2008), que las fallas de mercado desde el punto de vista de la demanda podrían ser compensadas por un mercado privado eficiente en la oferta de financiamiento. Sin embargo, los riesgos asumidos por educarse y la oferta de financiamiento no se logran equilibrar con la tasa de interés del mercado.

Stiglitz y Weiss (1981) señalan fenómenos de selección adversa y de riesgo moral en el financiamiento de ES. Estos fenómenos se presentan en el mercado del financiamiento de la educación porque los estudiantes que solicitan créditos tienen un mejor conocimiento de sus capacidades respecto de los prestamistas. Dada esta asimetría en la información, los prestamistas -al no observar las características de los estudiantes- fijan tasas similares a estudiantes con características distintas, y sólo fundándose en el promedio de la población estudiantil (en realidad, deberían hacerlo en función de las características "peores" de esta población, no sobre el promedio).

El prestamista desconoce si el estudiante se esfuerza y, de esta forma, tener un mejor desempeño en el mercado laboral que asegure que pagará la deuda adquirida. En consecuencia, el problema de riesgo moral se origina porque los términos de los créditos no pueden escribirse en función de los esfuerzos realizados por los estudiantes. La población estudiantil más dispuesta a aceptar los créditos con tasas altas es la que hace menos esfuerzo y la que tiene una probabilidad más baja de reembolsar el préstamo. Por lo tanto, los prestamistas tienden a incrementar las exigencias en términos de los requisitos 
solicitados (codeudores, fiadores), lo que acentúa que las personas de bajos ingresos queden por fuera del sistema educativo.

Desde el punto de vista teórico, el modelo tiene en cuenta que la educación no es un bien público "puro", por no cumplir con la propiedad de no exclusión, lo que justifica el financiamiento y provisión privada. Al plantearse la provisión pública del servicio de educación se le exigen rendimientos decrecientes a escala relacionados con una curva de costos medios decrecientes en el tramo relevante de la demanda, asimilable a servicios públicos caracterizados por una gran inversión inicial en capital fijo y redes de distribución para su cuasi universalización con bajos costos incrementales. Es decir, el agente maximizador se enfrentará al siguiente dilema:

$$
\begin{aligned}
& \underset{\text { educación }}{\operatorname{Max}} U_{i}(\text { educación, } \mathbf{n}) \forall i=1, \mathbf{n}, n \\
& \text { s.a. } \sum_{i=1}^{n} p_{i} X_{i}=m \wedge G(c)
\end{aligned}
$$

En el cual se supone que el individuo sigue las estructuras de preferencias de la teoría del consumidor. Ello garantiza la existencia de una función de utilidad, la que cumplirá, entre otros supuestos, con la no sociabilidad local para cada uno de los elementos que le otorgan utilidad, en especial de la educación. Dado el interés particular del presente estudio, se supone, ceteris paribus, que la utilidad depende de un conjunto finito de bienes en la cual sólo la educación varía. La función $G(c)$ representa el acceso al crédito educativo, el cual estará determinado por un vector $c$ de características tanto del proveedor del crédito como del individuo. Cada consumidor tiene previsión perfecta que derivaría una utilidad instantánea del consumo en cada instante del tiempo.

El objetivo del agente representativo sería maximizar la suma de la utilidad instantánea a lo largo de su ciclo de vida, descontada por un factor de descuento $\beta$.

$$
\sum_{z=1}^{T+T^{R}} B^{z-1} \frac{\left(C_{t-z-1}\right)^{1-\sigma}}{1-\sigma}
$$


Donde,

$\mathrm{T}+\mathrm{T}^{\mathrm{R}}$ : Tiempo final del agente representativo.

$\sigma$ : $\quad$ Representa el inverso de la elasticidad de sustitución intertemporal.

C: $\quad$ Consumo en educación.

El incentivo para dedicar tiempo al estudio y destinar parte del ingreso al gasto en educación consiste en incrementar el stock de capital humano y así obtener mayores ingresos durante el periodo de vida laboral. Se supone que se parte de una función con rendimientos constantes a escala.

Siguiendo a Leyva y Cárdenas (2002), tanto el aspecto de consumo como el de inversión de la educación rinden utilidad en el presente y en el futuro, por lo que ambos contribuyen a la corriente descontada de utilidad disfrutada por el sujeto económico. En este sentido, la educación difiere poco de cualquier otro bien duradero. En cambio, en otros aspectos tienen características diferentes:

- Los costos de la educación son prolongados.

- Es relativamente fácil medir el rendimiento de la inversión en educación. Excluyendo el elemento de consumo, es de esperar que, ceteris paribus, el valor actual descontado de los ingresos de toda la vida de un individuo con un alto nivel de educación superen los de una persona con un nivel de educación menor. Este diferencial proporciona una medida del rendimiento entre niveles educativos.

- Los beneficios de la educación son especialmente duraderos. Aun cuando la mayoría de los bienes se deprecian con el tiempo, los conocimientos y calificaciones no suelen hacerlo mientras se ejerciten regularmente.

Un riesgo común de estas tres características es la función que desempeña el tiempo. De acuerdo con Leyva y Cárdenas (2002), el mercado del servicio educativo no puede describirse adecuadamente a través de un modelo estático, en el que sólo se tenga en cuenta la utilidad y los costos actuales. En el modelo se debe suponer que la educación dura t años y que el individuo espera trabajar hasta el año T. El periodo base, para $\mathrm{i}=0$, se define como aquel en que se inicia 
la educación y formación. Entonces, el individuo invierte en capital humano hasta el punto en que se cumple para la unidad marginal de educación.

\subsection{Discusión del modelo econométrico ${ }^{19}$}

En cuanto a la aproximación empírica al modelo presentado, el individuo revelará su preferencia por la educación a través de las matrículas efectivas, las cuales representarán el resultado de una toma de decisión maximizadora por parte del agente. De este modo, la aproximación econométrica seguirá la siguiente forma funcional:

$$
\mathrm{M}_{\mathrm{it}}=\alpha+\mathrm{G}_{\mathrm{t}}+\mathrm{r}_{\mathrm{t}}+\mathrm{Yu}_{\mathrm{t}}+\mathrm{Ytec}_{\mathrm{t}}+\mathrm{U}_{\mathrm{it}}+\mathrm{S}_{\mathrm{t}}+\varepsilon_{\mathrm{iy}}+\mathrm{v}_{\mathrm{it}}
$$

Donde,

$\mathrm{M}_{\mathrm{it}}=$ Matrícula en educación superior en cada región i.

$G_{t}=$ Gasto público destinado a la oferta de educación superior.

$r_{t}=$ Tasa de interés promedio del crédito educativo.

$\mathrm{U}_{\mathrm{t}}=$ Tasa de desempleo en cada región i.

$\mathrm{X}_{\mathrm{t}}=$ Desembolsos en créditos educativos.

$\mathrm{Y}_{\mathrm{t}}=$ Ingreso técnico (YTEC) y universitario (YU).

$\mathrm{S}_{\mathrm{t}}=$ Solicitudes de créditos educativos. ${ }^{20}$

$\mathrm{YP}_{\mathrm{t}}^{2}=$ Ingreso per cápita regional.

El subíndice $(t)$ se refiere al intervalo del tiempo, en nuestro caso, anual para el periodo 1990 a 2005.

El subíndice (i) se refiere a los cross sections, en nuestro caso, tres de las principales ciudades del país (Bogotá, Medellín y Cali).

La tasa de matrícula en ES en cada región, $\mathrm{M}_{\mathrm{it}}$, se construyó a partir de la base de datos del ICFES para cada región de análisis.

19 La estimación del modelo se realizó con el paquete estadístico STATA 10.

20 El uso de esta variable puede traer algunas complicaciones, pues se podría argumentar que la variable de solicitud de créditos educativos tiene una alta endogeneidad en el modelo. Sin embargo, también podría decirse que no necesariamente es este el caso. Muchas de las solicitudes no resultan necesariamente en matrícula y por medio de esta variable se estaría capturando a la población que no tuvo acceso a ella y, a su vez, a aquellos a quienes se les niega el crédito y fueron matriculados. Agradecemos a uno de los evaluadores anónimos por este comentario. 
La tendencia de crecimiento de Medellín es superior a la del resto de las regiones, presentando altos crecimientos durante el periodo de análisis, a pesar de la disminución en 1993, 1997 y 2000. Cali presenta variaciones leves durante el periodo de análisis. Por su parte, Bogotá tiene un crecimiento alto entre 1996 y 1998, pero la tendencia se revierte e inicia un crecimiento menor en el número de matriculados.

El gasto público destinado a educación superior, $G_{t}$, se construyó a partir de los anuarios estadísticos municipales de cada región. La tasa de interés es un promedio de tasas de créditos educativos, recopilada de distintas instituciones de financiamiento a la educación. La variable tasa de desempleo se elaboró a partir de la información regional del Departamento Administrativo Nacional de Estadística (DANE). Los ingresos universitarios y técnicos se construyeron a partir de la información de la Encuesta Continua de Hogares (ECH), de 2000 a 2005, y como un promedio del ingreso regional desde 1990 hasta 1999. Finalmente, la variable solicitud de créditos educativos se elaboró a partir de información del ICFES.

\subsection{Resultados empíricos}

La estimación del modelo econométrico buscó establecer la significancia y los signos de algunas variables que miden el impacto del financiamiento en la demanda educativa, representado en el logaritmo de las matrículas de ES. El procedimiento econométrico sigue los desarrollos tradicionales de la técnica de Datos de Panel en la literatura (Baltagi, 2001); no obstante, la mayor disponibilidad de datos en el tiempo hace que la decisión sobre el método a estimar no se limite a los efectos no observados de cada individuo, sino también, a efectos temporales de las variables incluidas en el modelo. De este modo, se realizaron las pruebas de raíz unitaria para las diferentes especificaciones -con intercepto, con tendencia, entre otras- para panel bajo la hipótesis de homogeneidad en la raíz de los individuos del panel-Levin, Lin y Chu (LLC)-y heterogeneidad entre ellos -Im, Pesaran y Shin (IPS) ${ }^{21}$.

21 Para un desarrollo detallado de cada uno de estos contrastes, véase Baltagi (2001). 
Tabla 2. Pruebas de raíz unitaria*

\begin{tabular}{|c|c|c|c|c|c|c|c|}
\hline \multirow{2}{*}{ Contraste } & $M_{i t}$ & $i_{t}$ & $G_{t}$ & $U_{t}$ & $Y_{t}$ & $S_{t}$ & $Y_{t 2}$ \\
\hline \multirow{2}{*}{ LLC } & -2.264 & -6.689 & -3.288 & -0.660 & 0.318 & -1.896 & 5.389 \\
& $(0.98)$ & $(0.00)$ & $(0.00)$ & $(0.25)$ & $(0.62)$ & $(0.02)$ & $(1.00)$ \\
\hline \multirow{2}{*}{ IPS } & 1.8961 & -4.698 & -0.927 & -0.905 & 1.260 & -0.996 & 5.885 \\
& $(0.97)$ & $(0.00)$ & $(0.17)$ & $(0.18)$ & $(0.62)$ & $(0.16)$ & $(1.00)$ \\
\hline
\end{tabular}

* P-valor entre paréntesis.

Los resultados son sugerentes sobre la presencia de efectos temporales en la relación propuesta. A esto se suma la posibilidad de que los efectos no observados sean un proceso generador de endogeneidad. Bajo esta perspectiva, se realizó la prueba de raíz unitaria para las primeras diferencias de las series, lo que no permitió rechazar la existencia de estacionariedad en cada una de ellas. Al respecto, Wooldridge (2002) sugiere una solución si el efecto no observado está correlacionado con la matriz de datos. Piensa que el modelo se puede estimar por efectos fijos a través de las primeras diferencias de las series, recuperando la consistencia de los estimadores y desapareciendo los efectos temporales.

La inspección de no estacionariedad acompañada de la vista gráfica de las series revela la presencia de una tendencia determinística en la variable dependiente, lo que sugiere la existencia de efectos en el tiempo que incrementa el número de matriculados. Esto modifica la especificación inicial del modelo y hace necesario controlar esta tendencia dentro de las variables explicativas.

Gráfico 7. Tendencia determinística de las series del modelo

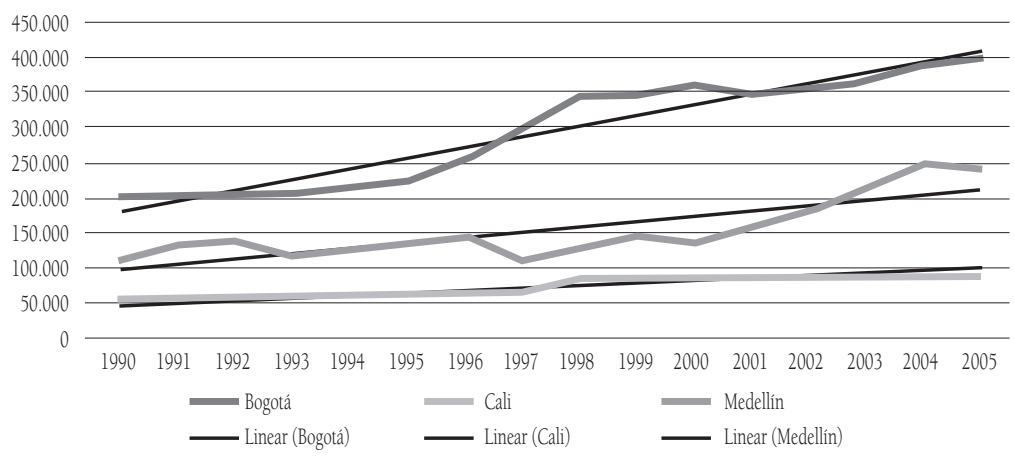

Fuente: Resultados del modelo. 
En el modelo los errores estándar son robustos para heterocedasticidad. A través de la aproximación del Contraste de Hausman, se verificó la no existencia de correlación entre los efectos no observados y la matriz de datos. De este modo, la manera de estimar el modelo es a través de efectos fijos para un panel balanceado. Como se describió, la muestra está compuesta por 16 observaciones en el tiempo para tres cross sections, con el fin de obtener en total 48 observaciones. Es decir, el intervalo de tiempo anual abarca el periodo de 1990 a 2005 y las tres cross sections: Bogotá, Medellín y Cali. En busca de encontrar la mejor especificación para el modelo se decide aplicar dos tipos de especificaciones: modelo individual por ciudad y panel. Esto permite contrastar la pertinencia de los resultados por municipio y luego su agregación.

A continuación se presentan los resultados del modelo para cada ciudad, intentando capturar efectos no observables o cambios estructurales. En el caso de Bogotá se encuentra: 1) la tasa de matrícula rezagada es significativa y tiene un efecto positivo sobre la variable dependiente, lo que concuerda con la tendencia determinística del modelo, 2) la variable ingresos de los técnicos no presenta relación positiva con la tasa de matrícula, 3) el ingreso no lineal (ingreso al cuadrado) presenta una relación positiva en el modelo, por lo que se establecería que el ingreso en Bogotá podría tener un efecto con rendimientos decrecientes, 4) incrementos en la tasa de interés generaría disminución en la tasa de matrículas en Bogotá, 5) mayor cantidad de solicitudes de créditos educativos generaría efectos positivos sobre la demanda educativa en Bogotá y 6) la tasa de desempleo no incidiría sobre la variable dependiente del modelo. 
Tabla 3. Demanda de la matrícula universitaria por ciudades (1990-2005) (Errores estándar en paréntesis)

\begin{tabular}{|c|c|c|c|}
\hline $\begin{array}{c}\text { Variable dependiente: } \\
\text { Matrícula universitaria }\end{array}$ & $\begin{array}{c}\text { Modelo Bogotá } \\
1990-2005\end{array}$ & $\begin{array}{c}\text { Modelo Cali } \\
1990-2005\end{array}$ & $\begin{array}{c}\text { Modelo Medellín } \\
1990-2005\end{array}$ \\
\hline M(-1) & $\begin{array}{c}1.396 \\
(11.075)\end{array}$ & NS & NS \\
\hline YTEC & $\begin{array}{c}-0.197 \\
(-2.359)\end{array}$ & NS & NS \\
\hline YP? & $\begin{array}{c}0.274 \\
(6.228)\end{array}$ & -0.157 & -0.131 \\
& -0.125 & -0.099 & $(-1.649)$ \\
\hline I? & $(-3.453707)$ & $(-2.477)$ & NS \\
\hline Tendencia determinística & 0.032 & 0.084 & 0.095 \\
\hline X? & $(2.156)$ & $(7.387)$ & $(4.962)$ \\
\hline R2 & 0.111 & -0.070 & 0.174 \\
& $(4.990)$ & $(-4.197)$ & $(2.654)$ \\
\hline DW & 0.997 & 0.994 & 0.901 \\
\hline
\end{tabular}

NS: variable no significativa.

Para el caso de Cali, el modelo presenta las siguientes relaciones. Por un lado, el desempleo no incidiría en el acceso educación. Los incentivos salariales no generarían mayor demanda educativa y esto se reflejaría con el signo negativo en el modelo. Mayor cantidad de solicitudes de créditos educativos no generaría efectos positivos sobre la demanda educativa. En el caso de Medellín, la demanda de matrículas universitarias dependería, desde las variables de análisis, sólo del incremento de las solicitudes y de la tendencia determinística (media en función del tiempo). Los resultados para Medellín muestran que ni la tasa de interés ni la tasa de desempleo ni los ingresos inciden sobre la dinámica de la ES.

Aunque los resultados de los modelos regionales no son contundentes, sobre la base de las pruebas realizadas se encuentra que la variable dependiente tiene tendencia determinística. La implicación es que cualquier choque que experimente la variable dependiente se revertirá en el largo plazo, aun cuando el impacto sea muy lento. Este resultado se encontró también en el modelo completo (ver tabla 3), en el cual la tendencia determinística es positiva, aunque el coeficiente sea muy pequeño. 
Los resultados muestran que incrementos de la tasa de interés promedio de los créditos educativos afectan la demanda de la matrícula universitaria en ES en las ciudades en análisis. Además, los ingresos afectarían positivamente la demanda universitaria en ES.

Tabla 4. Demanda de la matrícula universitaria 1990-2005

(Errores estándar en paréntesis)

\begin{tabular}{|c|c|}
\hline Variable dependiente: Matrícula universitaria & Modelo Panel (Efectos fijos) \\
\hline \multirow{2}{*}{ YTEC? } & 0.202998 \\
& $(1.907466)$ \\
\hline \multirow{2}{*}{ YP? } & -0.132505 \\
& $(-2.213803)$ \\
\hline \multirow{2}{*}{ ? } & -0.159082 \\
& $(-2.293635)$ \\
\hline \multirow{2}{*}{ Tendencia determinística } & 0.060155 \\
& $(2.658748)$ \\
\hline \multirow{2}{*}{ M(-1) } & 0.646956 \\
& $(5.312600)$ \\
\hline F & 0.000000 \\
R cuadrado & 0.988280 \\
DW & 1.849941 \\
\hline
\end{tabular}

Este modelo se contrasta con los resultados obtenidos por Albano (2005) para el caso de Argentina. En dicho modelo se establece para la demanda de capital humano una relación positiva con el diferencial de salarios y una negativa con los costos de la educación. Según este estudio, los factores determinantes de la matrícula en términos agregados podrían agruparse en cuatro aspectos: 1) el retorno de la inversión misma, 2) las características socioeconómicas de la región, 3) los factores propios de los mercados de trabajo regionales y 4) los factores relacionados con el nivel educativo de la población y con características propias de los subsistemas universitarios regionales.

Para el caso colombiano se encuentran características similares, ya que los ingresos resultan relevantes para dinamizar la matrícula universitaria (se podría pensar que desde el retorno de la inversión misma y por la capacidad de demanda de los agentes). En este caso, se encuentra que la tasa de interés tiene un efecto adverso para el crecimiento de la tasa de matrícula y la tendencia determinística es significativa y tiene un efecto positivo en la variable dependiente. 


\section{Conclusiones}

En este estudio el objetivo fue identificar la relación entre la demanda por ES y variables relacionadas con su financiamiento. En particular, se analizó como el financiamiento público y privado y las señales del mercado laboral explican las matrículas en ES en tres regiones de Colombia: Bogotá, Cali y Medellín.

En particular, y reconociendo las limitaciones que ello implica ${ }^{22}$, se identificaron variables macroeconómicas y financieras que pueden actuar como determinantes del acceso a la matrícula estudiantil. Los resultados han indicado que el financiamiento, tanto público como privado, de la educación determina la demanda por educación. Esto muestra de que faltan condiciones o incentivos para que las personas, especialmente jóvenes, accedan a la ES y, en este sentido, el financiamiento ha jugado un papel fundamental para el desarrollo intelectual bien sea público o privado.

La oferta de créditos educativos por parte de las entidades financieras es reducida, de corto plazo y con exigencias altas en cuanto a las garantías de codeudores y el nivel de ingresos. Este mercado financiero tiene consideraciones de rentabilidad, riesgo, plazo y garantía que no cumplen una función social y que, por lo tanto, no llegan a poblaciones de estratos más bajos del país. Los problemas más severos en el campo del financiamiento educativo en Colombia parten del hecho de que el gasto público en ES se reduce constantemente; además, el presupuesto se destina fundamentalmente al funcionamiento en detrimento del gasto en inversión; existe dependencia excesiva de una sola fuente de financiamiento. Las instituciones públicas dependen de los subsidios gubernamentales, mientras que las privadas de la ausencia de criterios de eficiencia institucional que determinen el monto del presupuesto.

La educación es una inversión que da cuenta de gran parte de las mejoras en la calidad de la población. Las personas necesitan

22 Una de las mayores limitaciones es no poder separar concretamente la población potencial que accede a las IES de la que por diferentes motivos no lo hace (efecto demanda), y separarla también de que exista mayor oferta educativa. Agradecemos este comentario a uno de los evaluadores externos. 
capacitarse para mejorar su entorno productivo. En este sentido, requiere un esfuerzo intensivo para transmitir las destrezas productivas pertinentes. Para el mercado laboral se necesitan jóvenes que cuenten con una educación y capacitación adecuada al incorporarse a la población económicamente activa. Cuando hay adelantos en el conocimiento aumentan los recursos productivos, mejora el nivel de vida y son fuente importante de ingresos y riqueza. La profundización financiera conduce a la canalización de recursos hacia los individuos que tengan incentivos, por las condiciones del mercado, en invertir en capital humano, lo que incide en el bienestar social.

El modelo da cuenta de la importancia de la tasa de interés, gasto público y crédito y financiamiento para explicar la demanda educativa en Bogotá, Medellín y Cali. Adicionalmente, existen otros determinantes que inciden en el acceso, como la tasa de desempleo y los ingresos de las personas que tienen ES.

Faltan opciones de financiamiento de largo plazo y un sistema de crédito educativo con diferentes modalidades que se adapten a las demandas de los estudiantes y sus familias. Además, es necesario avanzar en un proceso de reformas estructurales en el sistema de crédito a la ES, que incluya el fortalecimiento institucional de las entidades, esquemas que garanticen el repago de la cartera y un mecanismo de fondeo que permita obtener la mayor rentabilidad social del capital asignado a la educación.

De este trabajo surge una nueva pregunta: el aumento de créditos educativos ayuda a mejorar la demanda por educación, pero, ¿cuál es la incidencia de estos créditos en la sostenibilidad de los estudiantes hasta lograr culminar sus estudios profesionales sin problemas de deserción o rezago?

\section{Referencias bibliográficas}

Albano, Julieta (2005) Determinantes de la matrícula universitaria: una aplicación de la teoría del capital humano al caso argentino. La Plata: Universidad Nacional de La Plata. Departamento de Economía.

Baltagi, B. H. (2001) Econometric Analysis of Panel Data. Chichester, U.K: John Wiley and Sons. 
Barro, R. \& Lee, J.W. (1993) International Comparisons of Educational Attainment. Journal of Monetary Economics, 32, pp. 363-394.

Barro, R. (1997) Determinants of Economic Growth: a Cross-Country Study. Cambridge, U.S.A.: MIT Press.

Benhabib, J. \& Spiegel, M. (1994) The Role of Human Capital in Economic Development: Evidence from Aggregate Cross-country Data. Journal of Monetary Economics, 43, pp. 143-174.

Canton, E. y F. De Jong (2005) The Demand for Higher Education in the Netherlands, 1950-1999. Economics of Education Review, 24, pp. 651663.

Easterlin, R. (1981) Why isn't the Whole World Developed? Journal of Economic History, 41, pp. 1-19.

Forero R. Nohora y Bardey David (2008) Teorías y algunas experiencias internacionales en el financiamiento de la educación superior: lecciones para Colombia. Serie documentos de trabajo No. 37. Bogotá, Colombia: Universidad del Rosario.

Ionescu, Anamaria Fenicia (2006) The effect of the federal student loan program on college enrollment and default rates. Colgate University. October.

Kapur, D. and Crowley M. (2008) Beyond the ABCs: Higher Education and Developing Countries. Center for Global Development, working paper.

Krueger, A. \& Lindahl, M. (2001) Education for Growth: Why and for Whom? Journal of Economic Literature, 39, pp. 1101-1136.

Leyva, Soraya y Cárdenas, Antonio (2002) Economía de la educación: capital humano y rendimiento educativo. México: Universidad Autónoma Metropolitana.

McPherson, Michael S. and Shapiro, Morton Owen (2006) U.S Higher Education Finance. Handbook of the Economics of Education, 2, pp. 1404-1434.

Ministerio de Educación Nacional (2002) Estadísticas de la Educación Superior. Resumen Anual. Disponible en: http://www.mineducacion.gov. co/1621/articles-85665_archivo_pdfl.pdf, enero 20 de 2008.

Sánchez, T. Fabio (2008) Impacto del crédito educativo sobre el acceso con equidad y permanencia en el sistema de Educación Superior. Bogotá: Facultad de Economía, Centro de Estudios sobre Desarrollo Económico (CEDE), Universidad de los Andes.

Stevens, P and Weale, M. (2003) Education and Economic Growth. National Institute of Economic and Social Research, Working Paper. 
134 EDUCACIÓN SUPERIOR EN COLOMBIA (periodo 1990-2005) - Isabel Ruiz, Carlos Andrés Cano, Isabel Cristina Montes

Stigliz, J. and Weiss A. (1981) Credit Rationings in Markets with Imperfect Information. American Economic Review, 71, pp. 393-410.

Temple, J. (2001) Generalizations that aren't? Evidence on Education and Growth. European Economic Review, 45, pp. 905-918.

Wooldridge, J. (2002) Econometric analysis of cross section and panel data. Cambridge: MIT Press.

Recibido: 14 de agosto de 2008 Aceptado: 28 de octubre de 2008 


\section{Anexos}

Tabla 1A: Crédito ACCES

\begin{tabular}{|c|c|}
\hline Año & Número de créditos nuevos por semestre \\
\hline $2003-1$ & 9.410 \\
\hline $2003-2$ & 11.929 \\
\hline $2004-1$ & 11.077 \\
\hline $2004-2$ & 9.454 \\
\hline $2005-1$ & 8.446 \\
\hline $2005-2$ & 9.745 \\
\hline $2006-1$ & 10.741 \\
\hline $2006-2$ & 16.666 \\
\hline $2007-1$ & 25.208 \\
\hline $2007-2$ & 9.463 \\
\hline Total & 122.139 \\
\hline
\end{tabular}

Fuente: Sánchez, 2008. ICETEX, Crédito Acces.

Tabla 2A: Número de estudiantes matriculados en Bogotá, Antioquia y Valle del Cauca que accedieron a un crédito del ICETEX

\begin{tabular}{|c|c|c|c|c|c|c|c|c|c|c|}
\hline Año & Ninguno & $\%$ & 1 & $\%$ & 2 & $\%$ & 3 & $\%$ & 4 o más & $\%$ \\
\hline $1998-1$ & 23955 & $93 \%$ & 923 & $4 \%$ & 329 & $1,3 \%$ & 209 & $0,8 \%$ & 258 & $1,0 \%$ \\
\hline $1998-2$ & 37193 & $92 \%$ & 1534 & $4 \%$ & 580 & $1,4 \%$ & 414 & $1,0 \%$ & 497 & $1,2 \%$ \\
\hline $1999-1$ & 62835 & $92 \%$ & 2400 & $4 \%$ & 786 & $1,2 \%$ & 877 & $1,3 \%$ & 1163 & $1,7 \%$ \\
\hline $1999-2$ & 72709 & $92 \%$ & 2571 & $3 \%$ & 851 & $1,1 \%$ & 1004 & $1,3 \%$ & 1669 & $2,1 \%$ \\
\hline $2000-1$ & 94602 & $92 \%$ & 3094 & $3 \%$ & 1002 & $1,0 \%$ & 1131 & $1,1 \%$ & 2881 & $2,8 \%$ \\
\hline $2000-2$ & 106566 & $92 \%$ & 3225 & $3 \%$ & 1091 & $0,9 \%$ & 1158 & $1,0 \%$ & 3569 & $3,1 \%$ \\
\hline $2001-1$ & 131170 & $92 \%$ & 3635 & $3 \%$ & 1324 & $0,9 \%$ & 1306 & $0,9 \%$ & 4651 & $3,3 \%$ \\
\hline $2001-2$ & 141577 & $92 \%$ & 3888 & $3 \%$ & 1403 & $0,9 \%$ & 1346 & $0,9 \%$ & 5370 & $3,5 \%$ \\
\hline $2002-1$ & 166359 & $92 \%$ & 4494 & $2 \%$ & 1669 & $0,9 \%$ & 1517 & $0,8 \%$ & 6502 & $3,6 \%$ \\
\hline $2002-2$ & 177925 & $92 \%$ & 4614 & $2 \%$ & 1773 & $0,9 \%$ & 1566 & $0,8 \%$ & 7029 & $3,6 \%$ \\
\hline $2003-1$ & 193236 & $93 \%$ & 4716 & $2 \%$ & 1979 & $0,9 \%$ & 1573 & $0,8 \%$ & 7055 & $3,4 \%$ \\
\hline $2003-2$ & 204645 & $93 \%$ & 4857 & $2 \%$ & 2027 & $0,9 \%$ & 1430 & $0,7 \%$ & 6986 & $3,2 \%$ \\
\hline $2004-1$ & 215744 & $93 \%$ & 6947 & $3 \%$ & 1818 & $0,8 \%$ & 1031 & $0,4 \%$ & 6781 & $2,9 \%$ \\
\hline $2004-2$ & 225783 & $93 \%$ & 8092 & $3 \%$ & 1717 & $0,7 \%$ & 802 & $0,3 \%$ & 6374 & $2,6 \%$ \\
\hline $2005-1$ & 202180 & $93 \%$ & 8216 & $4 \%$ & 1325 & $0,6 \%$ & 646 & $0,3 \%$ & 4340 & $2,0 \%$ \\
\hline $2005-2$ & 209781 & $93 \%$ & 9250 & $4 \%$ & 1366 & $0,6 \%$ & 682 & $0,3 \%$ & 3630 & $1,6 \%$ \\
\hline $2006-1$ & 231293 & $93 \%$ & 10890 & $4 \%$ & 1591 & $0,6 \%$ & 672 & $0,3 \%$ & 2956 & $1,2 \%$ \\
\hline $2006-2$ & 241078 & $94 \%$ & 11882 & $5 \%$ & 1463 & $0,6 \%$ & 662 & $0,3 \%$ & 2346 & $0,9 \%$ \\
\hline $2007-1$ & 193499 & $95 \%$ & 6750 & $3 \%$ & 989 & $0,5 \%$ & 502 & $0,2 \%$ & 1187 & $0,6 \%$ \\
\hline
\end{tabular}

Fuente: Ministerio de Educación. Sistema para la Prevención y Análisis de la Deserción en las Instituciones de Educación Superior -SPADIES-. 
136 EDUCACIÓN SUPERIOR EN COLOMBIA (periodo 1990-2005) - Isabel Ruiz, Carlos Andrés Cano, Isabel Cristina Montes

Tabla 3A. Evolución de la matrícula universitaria en Colombia (1990-2005)

\begin{tabular}{|c|c|c|c|c|c|c|c|c|}
\hline Año & Medellín & $\%(1)$ & Bogotá & $\%(2)$ & Cali & $\%(3)$ & Nacional & Total (1)+(2)+(3) \\
\hline 1990 & 109887 & 22,4 & 199421 & 40,9 & 48812 & 10 & 487448 & 73,3 \\
\hline 1991 & 128123 & 25,1 & 201562 & 39,5 & 49520 & 9,7 & 510649 & 74,3 \\
\hline 1992 & 134526 & 25,1 & 205486 & 38,4 & 51205 & 9,6 & 535320 & 73,1 \\
\hline 1993 & 114831 & 21 & 207452 & 37,9 & 54620 & 10 & 547288 & 68,9 \\
\hline 1994 & 125639 & 21,8 & 215464 & 37,4 & 56944 & 9,9 & 576570 & 69,1 \\
\hline 1995 & 136656 & 21,2 & 222125 & 34,5 & 59450 & 9,2 & 644188 & 64,9 \\
\hline 1996 & 143092 & 21,3 & 254852 & 37,8 & 61065 & 9,1 & 673353 & 68,2 \\
\hline 1997 & 110063 & 14,3 & 301245 & 39 & 64520 & 8,4 & 772291 & 61,7 \\
\hline 1998 & 128102 & 14,6 & 346120 & 39,3 & 76925 & 8,7 & 879840 & 62,6 \\
\hline 1999 & 143441 & 16,3 & 346528 & 39,5 & 76650 & 8,7 & 877944 & 64,5 \\
\hline 2000 & 133328 & 14,3 & 358580 & 38,4 & 81112 & 8,7 & 934085 & 61,4 \\
\hline 2001 & 152541 & 15,6 & 345478 & 35,4 & 84490 & 8,6 & 977243 & 59,6 \\
\hline 2002 & 178938 & 16,8 & 354622 & 33,3 & 86428 & 8,1 & 1063786 & 58,2 \\
\hline 2003 & 209754 & 20,3 & 367450 & 35,5 & 92653 & 9 & 1035006 & 64,8 \\
\hline 2004 & 246463 & 22,3 & 389541 & 35,3 & 92027 & 8,3 & 1104051 & 65,9 \\
\hline 2005 & 243177 & 20,1 & 401568 & 33,1 & 100225 & 8,3 & 1212037 & 61,5 \\
\hline
\end{tabular}

Fuente: Instituto Colombiano para el Fomento de la Educación Superior ICFES. Elaboración propia. Datos en miles de personas. 